\title{
OPEN Significance of inflammatory indexes in atezolizumab monotherapy outcomes in previously treated non-small-cell lung cancer patients
}

Yuki Katayama $^{1}$, Tadaaki Yamada ${ }^{1 凶}$, Yusuke Chihara ${ }^{2}$, Satomi Tanaka ${ }^{1}$, Keiko Tanimura $^{1}$, Naoko Okura ${ }^{1}$, Kazuki Hirose ${ }^{3}$, Sayaka Uda ${ }^{4}$, Shinsuke Shiotsu ${ }^{4}$, Soichi Hirai ${ }^{5}$, Osamu Hiranuma ${ }^{5}$, Taishi Harada ${ }^{6}$, Takayuki Shimamoto $^{1}$, Masahiro Iwasaku ${ }^{1}$, Yoshiko Kaneko ${ }^{1}$, Junji Uchino ${ }^{1}$, Takayuki Takeda ${ }^{3} \&$ Koichi Takayama $^{1}$

Cancer immunotherapy, including atezolizumab monotherapy, is a promising alternative strategy for patients with advanced non-small-cell lung cancer (NSCLC). Several inflammatory indices have been reported as potential biomarkers regarding the effectiveness of various treatments. This study aimed to analyze the efficacy of atezolizumab monotherapy using baseline inflammatory markers in NSCLC patients. We retrospectively enrolled 81 NSCLC patients who received atezolizumab monotherapy at six different medical institutions in Japan. The Cox proportional hazards model was used to assess the impact of the clinical variables, including inflammatory indexes, on clinical outcomes. Median progression-free survival (PFS) and overall survival (OS) were 60 days and 252 days, respectively. The objective response rate was $7.4 \%$, and the disease control rate was $54.3 \%$. Patients with high neutrophil to lymphocyte ratio (NLR), low lymphocyte to monocyte ratio (LMR), and/or high platelet to lymphocyte ratio (PLR), at baseline, demonstrated substantially shorter PFS and OS compared to those with a low NLR, high LMR, and/or low PLR. The multivariate analysis demonstrated that a high baseline NLR was substantially associated with short PFS and short OS. Our retrospective observations suggest that inflammatory indices may be a potential negative prognostic factor of atezolizumab monotherapy outcomes in NSCLC patients.

Lung cancer is the leading cause of cancer death worldwide ${ }^{1}$. Immune checkpoint inhibitors (ICIs), which target the programmed cell death protein 1 (PD-1) and its ligand, programmed death-ligand 1 (PD-L1), have been approved in the United States, Japan, and other countries, for the treatment of non-small-cell lung cancer (NSCLC) patients. The PD-1 receptor is expressed on activated T cells and binds to PD-L1 and PD-L2 to avoid autoimmunity in peripheral tissues ${ }^{2}$. Clinically, the difference in blockade of PD-1 and PD-L1 is interesting. Treatment with monoclonal antibodies specific for PD-L1 can still permit binding between PD-1 and PD-L2, and result in reduced blockade of the negative inhibitory signals of the immune system in comparison to PD-1 antibodies. Further, a systematic review has demonstrated that PD-L1 inhibitors have a slightly lower incidence of grade $3 / 4$ immune-related pneumonitis as compared to PD-1 inhibitors ${ }^{3}$, while anti-PD-1 and anti-PD-L1 antibody monotherapy shows a similar clinical response in previously treated NSCLC patients ${ }^{4-7}$. Hence, investigations into the clinical biomarkers of effective anti-PD-L1 antibody treatment, which is a promising therapeutic strategy for NSCLC, are warranted.

\footnotetext{
${ }^{1}$ Department of Pulmonary Medicine, Graduate School of Medical Science, Kyoto Prefectural University of Medicine, 465, Kajii-cho, Kamigyo-ku, Kyoto 602-8566, Japan. 2Department of Respiratory Medicine, Uji-Tokushukai Medical Center, Uji, Japan. ${ }^{3}$ Department of Respiratory Medicine, Japanese Red Cross Kyoto Daini Hospital, Kyoto, Japan. ${ }^{4}$ Department of Respiratory Medicine, Japanese Red Cross Kyoto Daiichi Hospital, Kyoto, Japan. ${ }^{5}$ Department of Respiratory Medicine, Otsu City Hospital, Otsu, Japan. ${ }^{6}$ Department of Medical Oncology, Fukuchiyama City Hospital, Fukuchiyama, Japan. ${ }^{\circledR}$ email: tayamada@koto.kpu-m.ac.jp
} 
Atezolizumab is a humanized, engineered monoclonal antibody that targets PD-L1, and contributes to preventing the interaction between PD-L1 and B7.1 receptor. The OAK study, a randomized phase 3 trial, demonstrated that the atezolizumab treatment group had a median OS of 13.8 months, which was substantially higher than the 9.9 months observed for the docetaxel group. Moreover, atezolizumab monotherapy showed tolerability with a more favorable safety profile than docetaxel ${ }^{3,4}$. PD-L1 expression in tumors has been used clinically as a positive biomarker for effective ICI treatment in NSCLC ${ }^{5}$. However, the anti-PD-L1 antibody clone SP142, which was utilized for clinical trials with atezolizumab, was relatively less concordant in PD-L1 expression than other antibodies, such as $28-8,22 \mathrm{C} 3$, and SP263 in patients with NSCLC ${ }^{6,7}$. Furthermore, several recent studies have reported potential ICI biomarkers in the host, such as preexisting autoimmune antibodies ${ }^{8}$, steroid use ${ }^{9}$, microbiome ${ }^{10}$, white blood cell count ${ }^{11}$, sarcopenia ${ }^{12}$, and body mass index (BMI $)^{13-15}$. Several inflammatory indices, such as the NLR, LMR, and PLR, which are recognized as important markers of inflammatory processes, have also been reported as potential predictors of the effectiveness of anti-PD-1 antibody therapy ${ }^{16-18}$. However, little is known regarding the subpopulation of NSCLC patients who exhibit clinical outcomes that require treatment with atezolizumab monotherapy. In this retrospective study, we analyzed the efficacy of atezolizumab monotherapy, using the baseline values of specific inflammatory markers, in 81 patients previously treated for NSCLC.

\section{Results}

Patient characteristics. A total of 81 NSCLC patients, treated with atezolizumab between April 2018 and November 2019 at six different medical institutions in Japan, were enrolled in this study. The sample characteristics included a median age of 71 years (range 42-84), with 44 male patients (54.3\%), and 64 (79.0\%) patients with a history of smoking. The histological subtypes were 50 adenocarcinoma (61.7\%) and 17 squamous cell carcinomas $(21.0 \%)$. Metastatic disease was detected in the liver of 11 patients (13.6\%) and in the brain of 22 patients (27.2\%). With regards to disease staging, 19 patients (23.5\%) were at stage III, $51(63.0 \%)$ were at stage IV, and 11 (13.6\%) showed postoperative recurrence. An EGFR mutation was detected in 14 patients (17.3\%). There were no ALK positive patients. The ECOG-PS was $0-1$ for 64 of the patients $(79.0 \%)$ and $2-4$ for 17 patients (21.0\%). The PD-L1 TPS was $\geq 50 \%$ in 13 patients ( $16.0 \%), 1-49 \%$ in 24 patients $(29.6 \%)$, and $<0 \%$ in 28 patients (34.6\%) with 16 patients non-evaluable (19.8\%). The BMI was $\geq 25$ for 10 patients (12.3\%), 20-25 for 40 patients $(49.4 \%)$, and $<20$ for 31 patients $(38.3 \%)$. Furthermore, 14 patients $(17.2 \%)$ experienced an irAE of any grade. Atezolizumab treatment was administered as 2nd line therapy for 14 patients (17.2\%), 3rd line for 22 patients (27.2\%), and 4th line or later for 45 patients (55.6\%). Table 1 details the baseline characteristics of the patients enrolled in this study.

The roles of inflammatory indexes in atezolizumab treatment. Based on the RECIST criteria, with regards to atezolizumab treatment outcomes, no patients experienced a complete response ( $0 \%), 6$ experienced a partial response $(7.4 \%), 38$ were classified with a stable disease $(46.9 \%), 28$ met the criteria for progressive disease (34.6\%), and 9 were non-evaluable (11.1\%). The objective response rate was $7.4 \%$ (95\% CI $2.8 \%-15.4 \%$ ), and the disease control rate was $54.3 \%$ (95\% CI $42.9 \%-65.4 \%)$. The median PFS and OS were 60 days (95\% CI 49-86 days) and 252 days (95\% CI 197-NA days), respectively.

According to the log-rank test, patients with an NLR $>5$, LMR $\leq 1.5$, and PLR $>262$ demonstrated significantly shorter PFS than those with an NLR $\leq 5, \mathrm{LMR}>1.5$, and PLR $\leq 262$, respectively (42 days vs. 86 days, $\mathrm{p}<0.001 ; 37$ days vs. 84 days, $\mathrm{p}=0.0031 ; 48.5$ days vs. 90 days, $\mathrm{p}=0.033$, respectively) (Fig. $1 \mathrm{~A}-\mathrm{C}$ ). Additionally, patients with an NLR $>5, \operatorname{LMR} \leq 1.5$, and PLR $>262$ exhibited significantly shorter OS than those with an NLR $\leq 5$, $\mathrm{LMR}>1.5$, and PLR $\leq 262$, respectively (98 days vs. NA, $\mathrm{p}<0.001 ; 98$ days vs. 396 days, $\mathrm{p}<0.001 ; 106$ days vs. NA, $\mathrm{p}<0.001$, respectively) (Fig. 1D-F). Additionally, univariate analysis revealed that a neutrophil count $>4500 /$ $\mathrm{mm}^{3}$ (hazard ratio (HR): 1.62; 95\% CI 1.01-2.59; $\mathrm{p}=0.042$ ), lymphocyte count $>1000 / \mathrm{mm}^{3}$ (HR: $0.60 ; 95 \%$ CI 0.38-0.96; $\mathrm{p}=0.033$ ), NLR > 5 (HR: 2.47; 95\% CI 1.50-4.06: $\mathrm{p}<0.001$ ), LMR $\leq 1.5$ (HR: 0.48; 95\% CI 0.30-0.79; $\mathrm{p}=0.0040$ ), and PMR $>262$ (HR: 1.67; 95\% CI 1.04-2.68; $\mathrm{p}=0.035$ ) were significantly associated with PFS in patients receiving atezolizumab treatment. Further, univariate analysis of the patient data also revealed that neutrophil counts $>4500 / \mathrm{mm}^{3}$ (HR: 2.56; 95\% CI 1.38-4.74; $\left.\mathrm{p}=0.0028\right)$, lymphocyte counts $>1000 / \mathrm{mm}^{3}(\mathrm{HR}$ : 0.47; 95\% CI 0.26-0.87; $\mathrm{p}=0.015$ ), monocyte counts $>500 / \mathrm{mm}^{3}$ (HR: 1.96; 95\% CI 1.07-3.57; $\left.\mathrm{p}=0.029\right)$, NLR $>5$ (HR: 3.78; 95\% CI 2.04-7.04; p < 0.001), LMR $\leq 1.5$ (HR: 0.30; 95\% CI 0.17-0.55: p < 0.001), and PLR > 262 (HR: 2.82; 95\% CI 1.54-5.18; $\mathrm{p}<0.001$ ) were significantly associated with OS (Table 2).

Multivariate analysis included age, ECOG-PS, smoking history, NLR, albumin (Alb), and C-reactive protein (CRP), while excluding LMR, PLR, neutrophil count, and lymphocyte count to avoid multi-collinearity among the NLR, LMR, and PLR. Our multivariate analysis demonstrated that a high baseline NLR was independently associated with PFS (HR: 2.50; 95\% CI 1.40-4.56: $\mathrm{p}=0.0018$ ) and OS (HR: 2.91: 95\% CI 1.51-5.61; $\mathrm{p}=0.0014$ ) in patients receiving atezolizumab treatment (Table 3 ).

Clinical profiles associated with baseline NLR. Of the 81 patients, $31(38.3 \%)$ had a pretreatment NLR of $>5$ and the remaining 50 patients $(61.7 \%)$ had a pretreatment NLR of $\leq 5$. When comparing the clinical profiles of these two groups (Table 4), the continuous variables BMI, CRP, and Alb were found to be substantial prognostic factors in patients with pretreatment NLR $>5(\mathrm{p}=0.021, \mathrm{p}=0.0015$, and $\mathrm{p}<0.001$, respectively). According to the log-rank test, an additional analysis showed that the combination of NLR and CRP was substantially correlated with OS, which indicates the synergistic effect of the combined use of NLR and CRP as prognostic factors in NSCLC patients receiving atezolizumab monotherapy (Fig. 2). 


\begin{tabular}{|c|c|c|}
\hline Items & Group & n (\%) \\
\hline Age & Median (range) & $71(42-84)$ \\
\hline \multirow{2}{*}{ Gender } & Male & $44(54.3)$ \\
\hline & Female & $37(45.7)$ \\
\hline \multirow{4}{*}{ ECOG-PS } & 0 & $23(28.4)$ \\
\hline & 1 & $41(50.6)$ \\
\hline & 2 & $10(12.3)$ \\
\hline & 3 & $7(8.6)$ \\
\hline \multirow{3}{*}{ Histology } & Adenocarcinoma & $50(61.7)$ \\
\hline & Squamous cell carcinoma & $17(21.0)$ \\
\hline & Other & $14(17.3)$ \\
\hline \multirow{2}{*}{ Smoking status } & Never smoker & $17(21.0)$ \\
\hline & Current or former smoker & $64(79.0)$ \\
\hline \multirow{3}{*}{ Staging } & Stage III & $19(23.5)$ \\
\hline & Stage IV & $51(63.0)$ \\
\hline & Postoperative recurrence & $11(13.6)$ \\
\hline \multirow{2}{*}{ EGFR mutations } & Positive & $14(17.3)$ \\
\hline & Negative & $67(82.7)$ \\
\hline \multirow{4}{*}{ PD-L1 TPS } & $\geq 50 \%$ & $13(16.0)$ \\
\hline & $1-49 \%$ & $24(29.6)$ \\
\hline & $<1 \%$ & $28(34.6)$ \\
\hline & Not evaluation & $16(19.8)$ \\
\hline \multirow{2}{*}{ Metastasis } & Liver metastasis & $11(13.6)$ \\
\hline & Brain metastasis & $22(27.2)$ \\
\hline \multirow{3}{*}{ BMI } & $\mathrm{BMI}>25$ & $10(12.3)$ \\
\hline & $25 \geq \mathrm{BMI}>20$ & $40(49.4)$ \\
\hline & $\mathrm{BMI} \leq 20$ & $31(38.3)$ \\
\hline \multirow{2}{*}{ Immune-related adverse events (irAE) } & Yes & $14(17.3)$ \\
\hline & No & $67(82.7)$ \\
\hline \multirow{3}{*}{ Treatment line } & 2nd & $14(17.3)$ \\
\hline & $3 \mathrm{rd}$ & $22(27.2)$ \\
\hline & $\geq 4$ th & $45(55.6)$ \\
\hline
\end{tabular}

Table 1. Patient characteristics at the baseline.

\section{Discussion}

Several studies have demonstrated that clinical characteristics, such as age, ECOG-PS, and smoking status, are negative biomarkers related to the clinical outcomes of anti-PD-1 antibody treatment in patients with NSCLC $^{16,17,19}$. In contrast, current clinically useful biomarkers have not been fully identified in predicting the efficacy of anti-PD-L1 antibody atezolizumab monotherapy. A pooled cohort analysis of clinical trials involving 1,489 NSCLC patients demonstrated the significance of the lung immune prognostic index, which is derived from scoring of the baseline LDH levels and NLR, in predicting survival and response outcomes of NSCLC patients treated with atezolizumab ${ }^{20}$. In the current retrospective analysis of 81 NSCLC patients who received atezolizumab monotherapy, the pretreatment inflammatory indices, more specifically high NLR, low LMR, and high PLR, were substantially associated with shorter PFS and OS.

Increasing evidence suggests that cancer-related inflammation plays an important role in tumor development. Peripheral blood leukocytes, including neutrophils and lymphocytes, are involved in the systemic inflammatory response, and participate in tumorigenesis and tumor progression. Previous studies have shown that high levels of neutrophils promote cancer cell proliferation, invasion, and metastasis, and induce resistance to cancer therapeutics ${ }^{21,22}$. Additionally, peripheral neutrophil counts reportedly correlate directly with the intratumoral neutrophil population ${ }^{23}$. In contrast, lymphocytes inhibit tumor growth and invasion through their cytolytic activity. In fact, the immune response to human cancer cells depends primarily on the level of total lymphocytes, which can be sharply reduced by systemic inflammation. Specifically, relative lymphocytopenia may reflect lower levels of CD4 + T cells, which impairs cancer immune surveillance and defense $\mathrm{e}^{21,24}$.

NLR is a marker of the systemic inflammatory response and reflects the balance between neutrophils and lymphocytes ${ }^{25,26}$. Pretreatment NLR is associated with the clinical outcomes of several therapeutic interventions in NSCLC patients, such as the response to platinum-based first-line chemotherapy in metastatic NSCLC patients, and the prognosis in operable NSCLC patients ${ }^{27,28}$. Moreover, inflammatory indices, including NLR, PLR, and LMR, are potential prognostic markers for lung cancer patients ${ }^{29-32}$. Our multivariate analysis demonstrated that high baseline NLR is an independent factor associated with poor PFS and OS. It is; however, unclear whether a high NLR is an effective prognostic factor in NSCLC patients receiving atezolizumab monotherapy. 
A
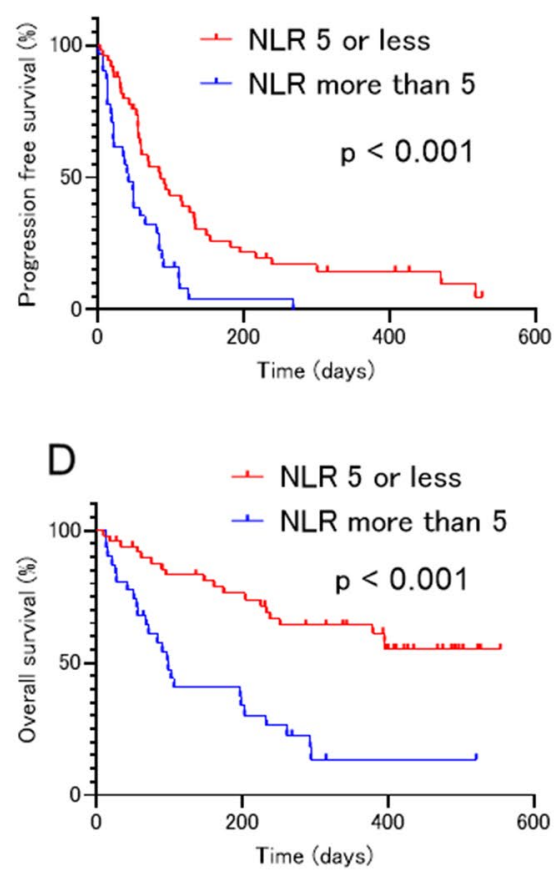

B

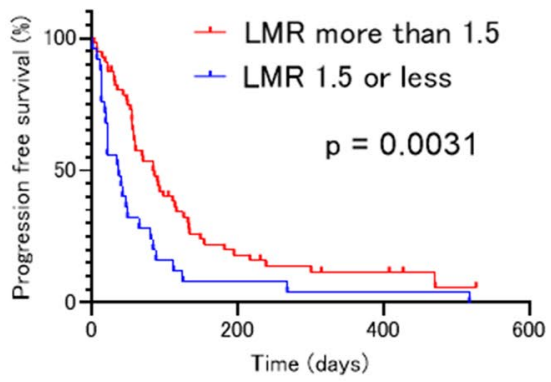

E

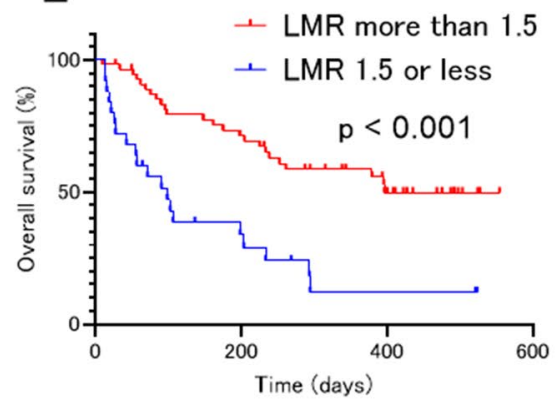

C

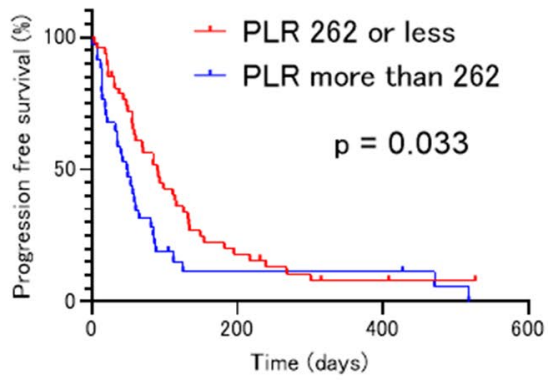

$\mathrm{F}$

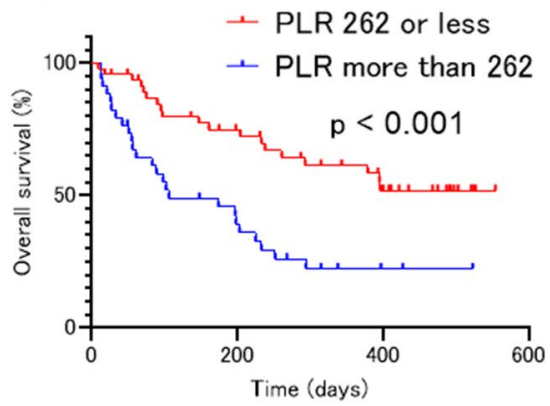

Figure 1. Kaplan-Meier survival curves for progression-free survival (PFS) and overall survival (OS). (A) The neutrophil to lymphocyte ratio (NLR) $>5$ (42 days vs. 86 days; $\mathrm{p}<0.001$ ), (B) the lymphocyte to monocyte ratio $(\mathrm{LMR}) \leq 1.5$ (37 days vs. 84 days; $\mathrm{p}=0.0031)$, and $(\mathrm{C})$ the platelet to lymphocyte ratio $(\mathrm{PLR})>262(48.5$ days vs. 90 days; $\mathrm{p}=0.033)$ were significantly associated with shorter PFS. (D) The neutrophil to lymphocyte ratio $(\mathrm{NLR})>5$ (98 days vs. NA; $\mathrm{p}<0.001$ ), (E) the lymphocyte to monocyte ratio (LMR) $\leq 1.5$ (98 days vs. 396 days; $\mathrm{p}<0.001$ ), and $(\mathbf{F})$ the platelet to lymphocyte ratio (PLR) $>262$ (106 days vs. NA; $\mathrm{p}<0.001)$ were significantly associated with shorter OS.

Therefore, it is necessary to further evaluate the clinical role of inflammatory scores, including NLR, in future studies.

As several independent clinical factors have indicated the disadvantages of immunotherapy in NSCLC patients, we evaluated the combined scores of several clinical characteristics. Our findings revealed that patients with high NLR are substantially correlated with several other clinical characteristics, such as low BMI, low Alb, and high CRP levels, at the baseline, compared to those with a low NLR. The present study is the first to reveal that the combination of a high baseline NLR and CRP levels is a potent prognostic factor for NSCLC patients receiving atezolizumab treatment. Moreover, persistent inflammatory responses in tumors suppress anti-tumor immunity and promote cancer progress through several mechanisms, including activation of type $2 \mathrm{~T}$ helper responses, which recruits regulatory $\mathrm{T}$ cells, and activation of the chemokine system ${ }^{33,34}$. Subsequently, neutrophils can be induced by cancer-associated inflammatory chemokines and cytokines, such as interleukin (IL)-6 and tumor necrosis factor ${ }^{35,36}$. CRP is also regulated by IL- 6 and IL- $1 \beta$, suggesting that induction of neutrophils and CRP may occur via similar inflammatory pathways. In fact, our results highlight an important relationship between high NLR and high CRP levels and promotion of poor prognosis. Although the usefulness of these combinatorial indexes remains largely unknown, they have the potential to serve as accurate biomarkers of cancer-related inflammation; hence, further large-scale investigations are warranted.

High plasma tumor mutational burden has been identified as a pivotal biomarker for the efficacy of atezolizumab monotherapy, and is associated with superior PFS in patients with previously treated NSCLC ${ }^{37}$; however, its associated cost makes in unfeasible for daily use. Alternatively, blood count analysis and CRP assessments are cost effective, form part of routine clinical practice, and reveal prognostic factors that could be useful in identifying NSCLC patients who will respond poorly to atezolizumab monotherapy, thereby assisting clinical decision-making regarding appropriate therapeutic interventions in previously treated NSCLC.

The present study has several limitations. Firstly, it is a retrospective study and the cohort had a limited sample size of 81 cases, even though treatment was administered in multiple medical institutions. Secondly, all patients in the cohort were Japanese. Thirdly, the study included several biases regarding patient conditions at commencement of atezolizumab therapy, such as the number of pretreatment regimens and the ECOG-PS of the patients. Finally, our findings revealed a substantial relationship between pre-treatment blood inflammatory markers and clinical outcomes, such as PFS and OS, in NSCLC patients treated with atezolizumab. These inflammatory markers might be a prognostic factor rather than a predictive factor for patients with this disease treated with atezolizumab. Although the current study was retrospective in nature, our novel biomarker findings 


\begin{tabular}{|l|l|c|l|c|}
\hline \multirow{2}{*}{ Items } & \multicolumn{2}{l|}{ PFS (univariate analysis) } & \multicolumn{2}{l|}{ OS (univariate analysis) } \\
\cline { 2 - 6 } & HR $(95 \%$ CI $)$ & p-value & HR $(95 \%$ CI) & p-value \\
\hline Age $\geq 75$ years & $0.74(0.43-1.25)$ & 0.25 & $1.14(0.60-2.15)$ & 0.69 \\
\hline Male gender & $1.76(1.08-2.85)$ & 0.022 & $1.48(0.81-2.71)$ & 0.20 \\
\hline Smoker & $1.02(0.58-1.81)$ & 0.94 & $1.59(0.71-3.57)$ & 0.26 \\
\hline ECOG-PS $\geq 2$ & $1.39(0.78-2.46)$ & 0.27 & $1.92(0.99-3.74)$ & 0.054 \\
\hline Squamous histology & $0.99(0.56-1.73)$ & 0.96 & $0.79(0.41-1.53)$ & 0.49 \\
\hline EGFR mutations positive & $1.52(0.83-2.78)$ & 0.18 & $1.24(0.55-2.79)$ & 0.61 \\
\hline Treatment line $\geq 4$ th & $1.11(0.70-1.77)$ & 0.66 & $1.84(0.99-3.41)$ & 0.053 \\
\hline BMI $>20$ & $0.76(0.47-1.22)$ & 0.25 & $0.47(0.26-0.85)$ & 0.012 \\
\hline BMI $>25$ & $0.88(0.42-1.85)$ & 0.74 & $0.56(0.20-1.56)$ & 0.26 \\
\hline Alb $>3.8$ g/dL & $0.68(0.41-1.13)$ & 0.136 & $0.52(0.27-1.01)$ & 0.0547 \\
\hline CRP $>0.89 \mathrm{mg} / \mathrm{dL}$ & $1.33(0.83-2.11)$ & 0.23 & $2.36(1.27-4.37)$ & 0.0064 \\
\hline LDH $>227$ U/L & $1.59(1.00-2.54)$ & 0.052 & $1.67(0.91-3.04)$ & 0.095 \\
\hline Neutrophil $>4500 / \mathrm{mm}^{3}$ & $1.62(1.01-2.59)$ & 0.042 & $2.56(1.38-4.74)$ & 0.0028 \\
\hline Lymphocyte $>1000 / \mathrm{mm}^{3}$ & $0.60(0.38-0.96)$ & 0.033 & $0.47(0.26-0.87)$ & 0.015 \\
\hline Monocyte $>500 / \mathrm{mm}^{3}$ & $1.53(0.96-2.44)$ & 0.072 & $1.96(1.07-3.57)$ & 0.029 \\
\hline Platelet $>250,000 / \mathrm{mm}^{3}$ & $1.29(0.80-2.08)$ & 0.29 & $1.31(0.72-2.38)$ & 0.37 \\
\hline NLR $>5.0$ & $2.47(1.50-4.06)$ & $<0.001$ & $3.78(2.04-7.04)$ & $<0.001$ \\
\hline LMR $>1.5$ & $0.48(0.30-0.79)$ & 0.0040 & $0.30(0.17-0.55)$ & $<0.001$ \\
\hline PLR $>262$ & $1.67(1.04-2.68)$ & 0.035 & $2.82(1.54-5.18)$ & $<0.001$ \\
\hline Liver metastasis & $1.85(0.96-3.54)$ & 0.064 & $1.55(0.69-3.48)$ & 0.29 \\
\hline Brain metastasis & $1.26(0.75-2.09)$ & 0.38 & $1.60(0.85-3.02)$ & 0.15 \\
\hline irAE & $1.81(0.92-3.56)$ & 0.09 & $1.23(0.52-2.91)$ & 0.64 \\
\hline PD-L1 TPS $1-49 \%(v s .<1 \%)$ & $1.46(0.86-2.49)$ & 0.16 & $1.31(0.65-2.64)$ & 0.46 \\
\hline PD-L1 TPS $\geq 50 \%(v s .0-49 \%)$ & $1.26(0.66-2.40)$ & 0.48 & $2.02(0.93-4.37)$ & 0.076 \\
\hline
\end{tabular}

Table 2. Cox proportional hazards and logistic regression models for progression free survival (PFS) and overall survival (OS).

\begin{tabular}{|l|l|l|l|l|}
\hline \multirow{2}{*}{ Items } & \multicolumn{2}{|l|}{ PFS (multivariate analysis) } & \multicolumn{2}{l|}{ OS (multivariate analysis) } \\
\cline { 2 - 5 } & HR $(\mathbf{9 5} \% \mathrm{CI})$ & $\boldsymbol{p}$-value & HR (95\%CI) & $\boldsymbol{p}$-value \\
\hline Age $>75$ years & $0.66(0.37-1.15)$ & 0.14 & $1.10(0.57-2.12)$ & 0.78 \\
\hline ECOG-PS $\geq 2$ & $1.39(0.75-2.58)$ & 0.073 & $1.63(0.81-3.30)$ & 0.17 \\
\hline Smoker & $0.98(0.54-1.80)$ & 0.93 & $1.28(0.56-2.92)$ & 0.56 \\
\hline NLR $>5.0$ & $2.50(1.40-4.56)$ & 0.0018 & $2.91(1.51-5.61)$ & 0.0014 \\
\hline Alb $>3.8 \mathrm{~g} / \mathrm{dL}$ & $0.92(0.53-1.60)$ & 0.76 & $0.80(0.40-1.62)$ & 0.54 \\
\hline $\mathrm{CRP}>0.89 \mathrm{mg} / \mathrm{dL}$ & $0.97(0.56-1.70)$ & 0.92 & $1.63(0.83-3.19)$ & 0.16 \\
\hline
\end{tabular}

Table 3. Cox proportional hazards and logistic regression models for progression free survival (PFS) and overall survival (OS) including the neutrophil to lymphocyte ratio (NLR).

regarding patient response to atezolizumab are notable and could be useful in addressing clinical issues. Future prospective investigations are necessary to verify our findings.

In summary, our observations showed that pretreatment inflammatory indexes, including a high NLR, could be promising negative prognostic factors for atezolizumab treatment in patients with previously treated for NSCLC. Since this retrospective study was conducted on a smaller scale, further experiments are needed to validate these observations.

\section{Methods}

Patients. We enrolled 81 patients, previously treated with chemotherapy for advanced NSCLC, who initiated atezolizumab monotherapy. The patients were treated between April 2018 and November 2019 at six different medical institutions, namely University Hospital, Kyoto Prefectural University of Medicine (Kyoto, Japan), Japanese Red Cross Kyoto Daiichi Hospital (Kyoto, Japan), Japanese Red Cross Kyoto Daini Hospital (Kyoto, Japan), Uji-Tokushukai Medical Center (Kyoto, Japan), Fukuchiyama City Hospital (Kyoto, Japan), and Otsu City Hospital (Shiga, Japan).

Atezolizumab was intravenously administered to patients as a fixed dose of $1200 \mathrm{mg}$ every three weeks. In general, these treatments continued until disease progression, intolerable toxicity, or patient refusal was noted. 


\begin{tabular}{|c|c|c|c|}
\hline Items & NLR > $5(n=31)$ & NLR $\leq 5(n=50)$ & $p$ value \\
\hline \multicolumn{4}{|l|}{ Age } \\
\hline Median (range) & $71(47-84)$ & $71(42-82)$ & 0.71 \\
\hline \multicolumn{4}{|l|}{ Gender } \\
\hline Male & 20 & 24 & 0.17 \\
\hline Female & 11 & 26 & \\
\hline \multicolumn{4}{|l|}{ Smoking status } \\
\hline Smoker & 26 & 38 & 0.58 \\
\hline Non-smoker & 5 & 12 & \\
\hline \multicolumn{4}{|l|}{ ECOG-PS } \\
\hline $0-1$ & 23 & 41 & 0.42 \\
\hline $2-4$ & 8 & 9 & \\
\hline \multicolumn{4}{|l|}{ Histology } \\
\hline $\mathrm{Sq}$ & 8 & 10 & 0.59 \\
\hline non-Sq & 23 & 40 & \\
\hline \multicolumn{4}{|c|}{ EGFR mutation status } \\
\hline Positive & 6 & 8 & 0.14 \\
\hline Negative & 25 & 42 & \\
\hline \multicolumn{4}{|l|}{ BMI } \\
\hline Median (range) & $19.2(12.7-26.6)$ & $21.3(14.7-24.4)$ & 0.021 \\
\hline \multicolumn{4}{|l|}{ Alb } \\
\hline Median (range) & $3.40(2.0-4.7)$ & $3.84(2.0-4.6)$ & 0.0015 \\
\hline \multicolumn{4}{|l|}{ CRP } \\
\hline Median (range) & $3.06(0.16-28.64)$ & $0.26(0.01-10.46)$ & $<0.001$ \\
\hline \multicolumn{4}{|l|}{ LDH } \\
\hline Median (range) & $250(156-794)$ & $223(143-1442)$ & 0.472 \\
\hline \multicolumn{4}{|l|}{ Liver metastasis } \\
\hline Positive & 5 & 6 & 0.741 \\
\hline Negative & 26 & 44 & \\
\hline \multicolumn{4}{|l|}{ Brain metastasis } \\
\hline Positive & 11 & 11 & 0.21 \\
\hline Negative & 20 & 39 & \\
\hline
\end{tabular}

Table 4. Patient characteristics related to baseline neutrophil to lymphocyte ratio (NLR) $(n=81)$.

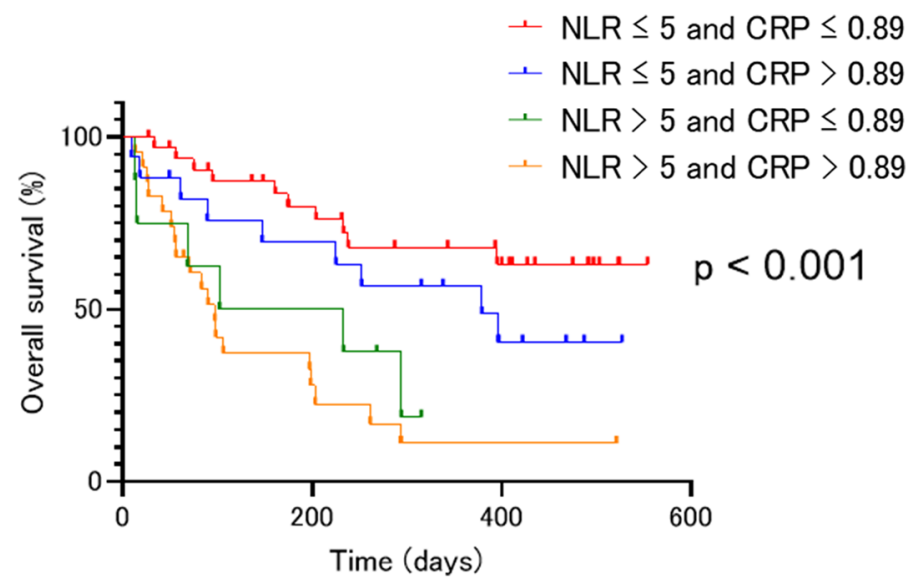

Figure 2. Kaplan-Meier survival curves for overall survival (OS) according to the combination of NLR and CRP levels. Overall survival (OS) was substantially longer in the group with a NLR $\leq 5$ and CRP $\leq 0.89 \mathrm{mg} / \mathrm{L}$ (NA) in comparison to the other groups with a NLR $\leq 5$ and CRP $>0.89 \mathrm{mg} / \mathrm{L}$ ( 379 days), an NLR $>5$ and $\mathrm{CRP} \leq 0.89 \mathrm{mg} / \mathrm{L}$ (167.5 days), and an NLR $>5$ and CRP $>0.89 \mathrm{mg} / \mathrm{L}(97$ days $)(\mathrm{p}<0.001)$. 
By the follow-up date, $72(88.9 \%)$ of the 81 patients had experienced progression of the disease, including 44 $(54.3 \%)$ that had passed away, while 37 patients (45.7\%) survived. The patients' clinical data, including age, sex, height, weight, BMI at the start of atezolizumab administration, histological subtype, PD-L1 expression level in tumors, epidermal growth factor receptor (EGFR) mutation status, anaplastic lymphoma kinase (ALK) fusion status, disease staging, metastatic site, corticosteroid administration, Eastern Cooperative Oncology Group Performance Status (ECOG-PS), smoking status, baseline laboratory findings, OS, PFS, as well as response rate and disease control rate, based on the Response Evaluation Criteria in Solid Tumors (RECIST; version 1.1), were retrospectively obtained from their medical records. The tumor-node-metastasis (TNM) stage was classified using the TNM stage classification system, version 8 . The study protocol was approved by the Ethics Committees of the Kyoto Prefectural University of Medicine and of each hospital. The work described herein has been carried out in accordance with the principles of the Declaration of Helsinki. Informed consent was obtained from all participants.

Tumor PD-L1 analysis. PD-L1 expression was analyzed by SRL, Inc. using a PD-L1 IHC 22C3 pharmDx assay (Agilent Technologies, Santa Clara, CA). The PD-L1 tumor proportion score (TPS) was calculated as a percentage in at least 100 viable tumor cells with complete, or partial, membrane staining. Pathologists at SRL, Inc. interpreted the TPS results.

Laboratory findings. The NLR, LMR, and PLR were defined as absolute neutrophil counts divided by absolute lymphocyte count, absolute lymphocyte count divided by absolute monocyte count, and absolute platelet count divided by absolute lymphocyte count, respectively. We measured baseline albumin (Alb), C-reactive protein (CRP), lactate dehydrogenase (LDH), as well as neutrophil, lymphocyte, monocyte, and platelet counts, and NLR, LMR, and PLR. Baseline was defined as day - 10 to 0 of the first atezolizumab administration. Cut off points of $N L R=5, L M R=1.5$, and $P L R=262$ were selected based on previous studies ${ }^{16,19}$. The cutoff values for baseline albumin, LDH, CRP, neutrophil, lymphocyte, monocyte, and platelets, were the respective median values.

Statistical analysis. Statistical analyses were performed using EZR statistical software, version $1.30^{38}$. All statistical tests carried out were two-sided and $\mathrm{p}<0.05$ was regarded as statistically significant. The PFS and OS were calculated using the Kaplan-Meier method, and differences were compared using the log-rank test. Continuous variables were analyzed using the Mann-Whitney $U$ test, while categorical variables were analyzed using Fisher's exact test. Univariate analyses were performed using the Cox proportional hazards and logistic regression models.

\section{Data availability}

The datasets generated during and/or analysed during the current study are available from the corresponding author on reasonable request.

Received: 30 May 2020; Accepted: 5 October 2020

Published online: 15 October 2020

\section{References}

1. Miller, K. D. et al. Cancer statistics for Hispanics/Latinos, 2018. CA Cancer J. Clin. 68, 425-445. https://doi.org/10.3322/caac.21494 (2018).

2. Pardoll, D. M. The blockade of immune checkpoints in cancer immunotherapy. Nat. Rev. Cancer 12, 252-264. https://doi. org/10.1038/nrc3239 (2012).

3. Rittmeyer, A. et al. Atezolizumab versus docetaxel in patients with previously treated non-small-cell lung cancer (OAK): a phase 3 , open-label, multicentre randomised controlled trial. Lancet 389, 255-265. https://doi.org/10.1016/s0140-6736(16)32517-x (2017).

4. Fehrenbacher, L. et al. Atezolizumab versus docetaxel for patients with previously treated non-small-cell lung cancer (POPLAR): a multicentre, open-label, phase 2 randomised controlled trial. Lancet 387, 1837-1846. https://doi.org/10.1016/s0140-6736(16)00587 -0 (2016).

5. Reck, M. et al. Pembrolizumab versus chemotherapy for PD-L1-positive non-small-cell lung cancer. N. Engl. J. Med. 375, 18231833. https://doi.org/10.1056/NEJMoa1606774 (2016).

6. Hirsch, F. R. et al. PD-L1 immunohistochemistry assays for lung cancer: results from phase 1 of the Blueprint PD-L1 IHC assay comparison project. J. Thorac. Oncol. 12, 208-222. https://doi.org/10.1016/j.jtho.2016.11.2228 (2017).

7. Fujimoto, D. et al. Predictive performance of four programmed cell death ligand 1 assay systems on nivolumab response in previously treated patients with non-small cell lung cancer. J. Thorac. Oncol. 13, 377-386. https://doi.org/10.1016/j.jtho.2017.11.123 (2018).

8. Toi, Y. et al. Profiling preexisting antibodies in patients treated with anti-PD-1 therapy for advanced non-small cell lung cancer. JAMA Oncol. 5, 376-383. https://doi.org/10.1001/jamaoncol.2018.5860 (2019).

9. Arbour, K. C. et al. Impact of baseline steroids on efficacy of programmed cell death-1 and programmed death-ligand 1 blockade in patients with non-small-cell lung cancer. J. Clin. Oncol. 36, 2872-2878. https://doi.org/10.1200/jco.2018.79.0006 (2018).

10. Katayama, Y. et al. The role of the gut microbiome on the efficacy of immune checkpoint inhibitors in Japanese responder patients with advanced non-small cell lung cancer. Transl. Lung Cancer Res. 8, 847-853. https://doi.org/10.21037/tlcr.2019.10.23 (2019).

11. Zer, A. et al. Correlation of neutrophil to lymphocyte ratio and absolute neutrophil count with outcomes with PD-1 axis inhibitors in patients with advanced non-small-cell lung cancer. Clin. Lung Cancer 19, 426-434.e421. https://doi.org/10.1016/j.cllc.2018.04.008 (2018).

12. Nishioka, N. et al. Association of sarcopenia with and efficacy of anti-PD-1/PD-L1 therapy in non-small-cell lung cancer. J. Clin. Med. https://doi.org/10.3390/jcm8040450 (2019).

13. McQuade, J. L. et al. Association of body-mass index and outcomes in patients with metastatic melanoma treated with targeted therapy, immunotherapy, or chemotherapy: a retrospective, multicohort analysis. Lancet Oncol. 19, 310-322. https://doi. org/10.1016/s1470-2045(18)30078-0 (2018). 
14. Cortellini, A. et al. A multicenter study of body mass index in cancer patients treated with anti-PD-1/PD-L1 immune checkpoint inhibitors: when overweight becomes favorable. J. Immunother. Cancer 7, 57. https://doi.org/10.1186/s40425-019-0527-y (2019).

15. Ichihara, E. et al. The impact of body mass index on the efficacy of anti-PD-1/PD-L1 antibodies in patients with non-small cell lung cancer. Lung Cancer 139, 140-145. https://doi.org/10.1016/j.lungcan.2019.11.011 (2020).

16. Diem, S. et al. Neutrophil-to-Lymphocyte ratio (NLR) and Platelet-to-Lymphocyte ratio (PLR) as prognostic markers in patients with non-small cell lung cancer (NSCLC) treated with nivolumab. Lung Cancer 111, 176-181. https://doi.org/10.1016/j.lungc an.2017.07.024 (2017).

17. Bagley, S. J. et al. Pretreatment neutrophil-to-lymphocyte ratio as a marker of outcomes in nivolumab-treated patients with advanced non-small-cell lung cancer. Lung Cancer 106, 1-7. https://doi.org/10.1016/j.lungcan.2017.01.013 (2017).

18. Ohba, T. et al. Prognostic impact of the Controlling Nutritional Status score in patients with non-small cell lung cancer treated with pembrolizumab. J. Thorac. Dis. 11, 3757-3768. https://doi.org/10.21037/jtd.2019.09.29 (2019).

19. Katayama, Y. et al. Impact of bowel movement condition on immune checkpoint inhibitor efficacy in patients with advanced nonsmall cell lung cancer. Thorac. Cancer 10, 526-532. https://doi.org/10.1111/1759-7714.12969 (2019).

20. Sorich, M. J., Rowland, A., Karapetis, C. S. \& Hopkins, A. M. Evaluation of the lung immune prognostic index for prediction of survival and response in patients treated with atezolizumab for NSCLC: pooled analysis of clinical trials. J. Thorac. Oncol. 14, 1440-1446. https://doi.org/10.1016/j.jtho.2019.04.006 (2019).

21. Diakos, C. I., Charles, K. A., McMillan, D. C. \& Clarke, S. J. Cancer-related inflammation and treatment effectiveness. Lancet Oncol. 15, e493-503. https://doi.org/10.1016/s1470-2045(14)70263-3 (2014).

22. Mantovani, A., Allavena, P., Sica, A. \& Balkwill, F. Cancer-related inflammation. Nature 454, 436-444. https://doi.org/10.1038/ nature07205 (2008).

23. Moses, K. \& Brandau, S. Human neutrophils: Their role in cancer and relation to myeloid-derived suppressor cells. Semin. Immunol. 28, 187-196. https://doi.org/10.1016/j.smim.2016.03.018 (2016).

24. Ferrone, C. \& Dranoff, G. Dual roles for immunity in gastrointestinal cancers. J. Clin. Oncol. 28, 4045-4051. https://doi.org/10.1200/ jco.2010.27.9992 (2010).

25. Forrest, L. M., McMillan, D. C., McArdle, C. S., Angerson, W. J. \& Dunlop, D. J. Evaluation of cumulative prognostic scores based on the systemic inflammatory response in patients with inoperable non-small-cell lung cancer. Br. J. Cancer 89, 1028-1030. https ://doi.org/10.1038/sj.bjc.6601242 (2003).

26. Takeda, T., Takeuchi, M., Saitoh, M. \& Takeda, S. Neutrophil-to-lymphocyte ratio after four weeks of nivolumab administration as a predictive marker in patients with pretreated non-small-cell lung cancer. Thorac. Cancer 9, 1291-1299. https://doi. org/10.1111/1759-7714.12838 (2018).

27. Liu, Z. L. et al. Neutrophil-lymphocyte ratio as a prognostic marker for chemotherapy in advanced lung cancer. Int. J. Biol. Markers 31, e395-e401. https://doi.org/10.5301/jbm.5000222 (2016).

28. Zhang, H. et al. Clinical significance of preoperative neutrophil-lymphocyte vs platelet-lymphocyte ratio in primary operable patients with non-small cell lung cancer. Am. J. Surg. 210, 526-535. https://doi.org/10.1016/j.amjsurg.2015.03.022 (2015).

29. Chen, Y. et al. Prognostic significance of combined preoperative platelet-to-lymphocyte ratio and lymphocyte-to-monocyte ratio in patients undergoing surgery with stage IB non-small-cell lung cancer. Cancer Manag. Res. 10, 5411-5422. https://doi.org/10.2147/ cmar.S177320 (2018).

30. Gao, Y. et al. Preoperative pulmonary function correlates with systemic inflammatory response and prognosis in patients with non-small cell lung cancer: results of a single-institution retrospective study. Oncotarget 8, 27489-27501. https://doi.org/10.18632 /oncotarget.14225 (2017).

31. Łochowski, M. et al. Prognostic value of neutrophil-to-lymphocyte, platelet-to-lymphocyte and lymphocyte-to-monocyte ratio ratios in patients operated on due to non-small cell lung cancer. J. Thorac. Dis. 11, 3377-3384. https://doi.org/10.21037/ jtd.2019.07.72 (2019).

32. Yang, J. et al. Pre-treatment inflammatory indexes as predictors of survival and cetuximab efficacy in metastatic colorectal cancer patients with wild-type RAS. Sci. Rep. 7, 17166. https://doi.org/10.1038/s41598-017-17130-6 (2017).

33. O'Callaghan, D. S., O'Donnell, D., O'Connell, F. \& O'Byrne, K. J. The role of inflammation in the pathogenesis of non-small cell lung cancer. J Thorac. Oncol. 5, 2024-2036. https://doi.org/10.1097/jto.0b013e3181f387e4 (2010).

34. Tauler, J. \& Mulshine, J. L. Lung cancer and inflammation: interaction of chemokines and hnRNPs. Curr. Opin. Pharmacol. 9, 384-388. https://doi.org/10.1016/j.coph.2009.06.004 (2009).

35. Ulich, T. R., del Castillo, J., Keys, M., Granger, G. A. \& Ni, R. X. Kinetics and mechanisms of recombinant human interleukin 1 and tumor necrosis factor-alpha-induced changes in circulating numbers of neutrophils and lymphocytes. J. Immunol. 139, 3406-3415 (1987).

36. Ulich, T. R., del Castillo, J., Guo, K. \& Souza, L. The hematologic effects of chronic administration of the monokines tumor necrosis factor, interleukin-1, and granulocyte-colony stimulating factor on bone marrow and circulation. Am. J. Pathol. 134, 149-159 (1989).

37. Gandara, D. R. et al. Blood-based tumor mutational burden as a predictor of clinical benefit in non-small-cell lung cancer patients treated with atezolizumab. Nat. Med. 24, 1441-1448. https://doi.org/10.1038/s41591-018-0134-3 (2018).

38. Kanda, Y. Investigation of the freely available easy-to-use software "EZR" for medical statistics. Bone Marrow Transplant. 48, 452-458. https://doi.org/10.1038/bmt.2012.244 (2013).

\section{Author contributions}

Y.K. and T.Y. of applicable authors had full access to all the data in the study and take responsibility for the integrity of the data and the accuracy of the data analysis. Conception and design: T.Y., T.T., and K.T. of applicable authors. Provision of study materials or patients: Y.K., T.Y., Y.C., S.T., K. Tanimura, N.O., K.H., S.U., S.S., S.H., O.H., T.H., T.S., M.I., Y.Kaneko, J.U., T.T., K.T. of applicable authors. Collection and assembly of data: Y.K., Y.C., S.T., K.Tanimura, N.O., K.H., S.U., S.S., S.H., O.H., T.H., T.T. of applicable authors. Data analysis and interpretation: Y.K., T.Y., M.I. of applicable authors. Manuscript writing: Y.K., T.Y., K.T. of applicable authors. Final approval of manuscript: All authors.

\section{Competing interests}

The authors declare no competing interests.

\section{Additional information}

Correspondence and requests for materials should be addressed to T.Y.

Reprints and permissions information is available at www.nature.com/reprints. 
Publisher's note Springer Nature remains neutral with regard to jurisdictional claims in published maps and institutional affiliations.

(c) (1) Open Access This article is licensed under a Creative Commons Attribution 4.0 International License, which permits use, sharing, adaptation, distribution and reproduction in any medium or format, as long as you give appropriate credit to the original author(s) and the source, provide a link to the Creative Commons licence, and indicate if changes were made. The images or other third party material in this article are included in the article's Creative Commons licence, unless indicated otherwise in a credit line to the material. If material is not included in the article's Creative Commons licence and your intended use is not permitted by statutory regulation or exceeds the permitted use, you will need to obtain permission directly from the copyright holder. To view a copy of this licence, visit http://creativecommons.org/licenses/by/4.0/.

(c) The Author(s) 2020 